\title{
Identification, synthesis, and conformation of tri- and tetrathiacycloalkanes from marine bacteria
}

Paul Sobik, Joerg Grunenberg, Katalin Böröczky, Hartmut Laatsch, Irene Wagner-Döbler, Stefan Schulz

\section{Table of Contents:}

$\begin{array}{ll}\text { Methods and Materials } & \text { S2 }\end{array}$

HRMS-data of the compounds A-E. S2

MS data of 4,4-dimethyl-1,2,3-trithiolane (1). S3

${ }^{1} \mathrm{H}-\mathrm{NMR}$ of 4,4,6,6-tetramethyl-1,2,5-trithiepane (2) at different temperatures. S4

MS of 4,4,6,6-tetramethyl-1,2,5-trithiepane (2). S5

${ }^{1} \mathrm{H}-\mathrm{NMR}$ of 3,3,7,7-tetramethyl-1,2,5-trithiepane (3) at different temperatures. S6

${ }^{1} \mathrm{H}$-NMR of 3,3,7,7-tetramethyl-1,2,5,6-tetrathiocane (5) at different temperatures.

MS of 3,3,7,7-tetramethyl-1,2,5,6-tetrathiocane (5). S8

${ }^{1} \mathrm{H}-\mathrm{NMR}$ of 3,3,8,8-tetramethyl-1,2,5,6-tetrathiocane (6) at different temperatures. S9

MS of 3,3,8,8-tetramethyl-1,2,5,6-tetrathiocane (6). S10

MS data of 5,5-dimethyl-1,2,3,4-tetrathiane (7). S10

${ }^{1}$ H-NMR of methyl-1,2-propanedithiol (8).
S11

MS of methyl-1,2-propanedithiol (8). $\quad$ S12

${ }^{1} \mathrm{H}$-NMR of 2,2-dimethylthiirane (10). $\quad$ S13

${ }^{1}$ H-NMR spectrum of 2-chlor-1-(2-chlor-2-methylpropyldisulfanyl)-2-methylpropane (11). $\quad$ S14

MS spectrum of 2-chlor-1-(2-chlor-2-methylpropyldisulfanyl)-2-methylpropane (11). $\quad$ S15

B3LYP 6-311G(d,p) optimized cartesian coordinates of all studied conformers for 3,3,6,6-

tetramethyl-1,2,5-trithiepane (4). 


\section{Methods and Materials.}

GC-MS analyses (EI, 70 eV) were performed using a GC-MSD system. A BPX-5 (25m x 0.25m x 0.25 $\mu \mathrm{m}$, SGE) capillary column with He as the carrier gas was used with a constant flow rate of $1 \mathrm{ml} / \mathrm{min}$. The initial oven temperature was $50^{\circ} \mathrm{C}$, held for 2 minutes, followed by heating with $3^{\circ} \mathrm{C} / \mathrm{min}$ to $320^{\circ} \mathrm{C}$. NMR spectra were recorded with tetramethylsilane as an internal standard. TLC analyses were performed on pre-coated silica gel sheets (Polygram ${ }^{\circledR}$ SIL G/UV 254 , Macherey-Nagel). For visualization 8 \% molybdatophosphoric acid in ethanol was used. Column chromatography was performed using silica gel (silica gel 60, corn size 0.069-0.200 mm, Merck). IR-data were obtained using a diamond-ATR-technique. 
TABLE S1. Elemental compositions and relative intensities of the fragments in the mass spectra of compounds A-E.
A
B
C
D
E

\begin{tabular}{|c|c|c|c|c|c|c|}
\hline Formula & $\mathrm{m} / \mathrm{z}$ & 1 RI:1175 & 3/4 RI:1472 & 2 RI:1516 & 6 RI:1727 & 5 RI:1748 \\
\hline $\mathrm{C}_{8} \mathrm{H}_{16} \mathrm{~S}_{4}^{+}$. & 240 & - & - & - & 39 & 62 \\
\hline $\mathrm{C}_{8} \mathrm{H}_{16} \mathrm{~S}_{3}{ }^{+}$ & 208 & - & 100 & 82 & - & - \\
\hline $\mathrm{C}_{4} \mathrm{H}_{8} \mathrm{~S}_{3}^{+}$ & 152 & 100 & 59 & 100 & 100 & 100 \\
\hline $\mathrm{C}_{8} \mathrm{H}_{15} \mathrm{~S}^{+}$ & 143 & - & 13 & - & - & - \\
\hline $\mathrm{C}_{4} \mathrm{H}_{8} \mathrm{~S}_{2}^{+}$ & 120 & - & 28 & 26 & 24 & 59 \\
\hline$\left(\mathrm{C}_{3} \mathrm{H}_{6} \mathrm{~S}_{2}\right)^{+}$ & 106 & 2 & 12 & 42 & 1 & - \\
\hline $\mathrm{S}_{3}^{+}$ & 96 & 4 & 1 & 1 & - & - \\
\hline $\mathrm{C}_{4} \mathrm{H}_{8} \mathrm{~S}^{+}$ & 88 & 27 & 53 & 41 & 24 & 26 \\
\hline $\mathrm{C}_{4} \mathrm{H}_{7} \mathrm{~S}^{+}$ & 87 & 52 & 94 & 87 & 56 & 55 \\
\hline $\mathrm{C}_{3} \mathrm{H}_{5} \mathrm{~S}^{+}$ & 73 & 10 & 10 & 8 & 2 & 19 \\
\hline $\mathrm{S}_{2}^{+}$ & 64 & 13 & 4 & 3 & 4 & 4 \\
\hline $\mathrm{C}_{2} \mathrm{H}_{3} \mathrm{~S}^{+}$ & 59 & 21 & 27 & 32 & 11 & 15 \\
\hline $\mathrm{C}_{4} \mathrm{H}_{7}^{+}$ & 55 & 38 & 95 & 76 & 54 & 82 \\
\hline $\mathrm{CHS}^{+}$ & 45 & 17 & 19 & 18 & 9 & 10 \\
\hline $\mathrm{C}_{3} \mathrm{H}_{5}^{+}$ & 41 & 19 & 27 & 29 & 17 & 23 \\
\hline $\mathrm{C}_{3} \mathrm{H}_{3}^{+}$ & 39 & 18 & 21 & 20 & 13 & 16 \\
\hline
\end{tabular}




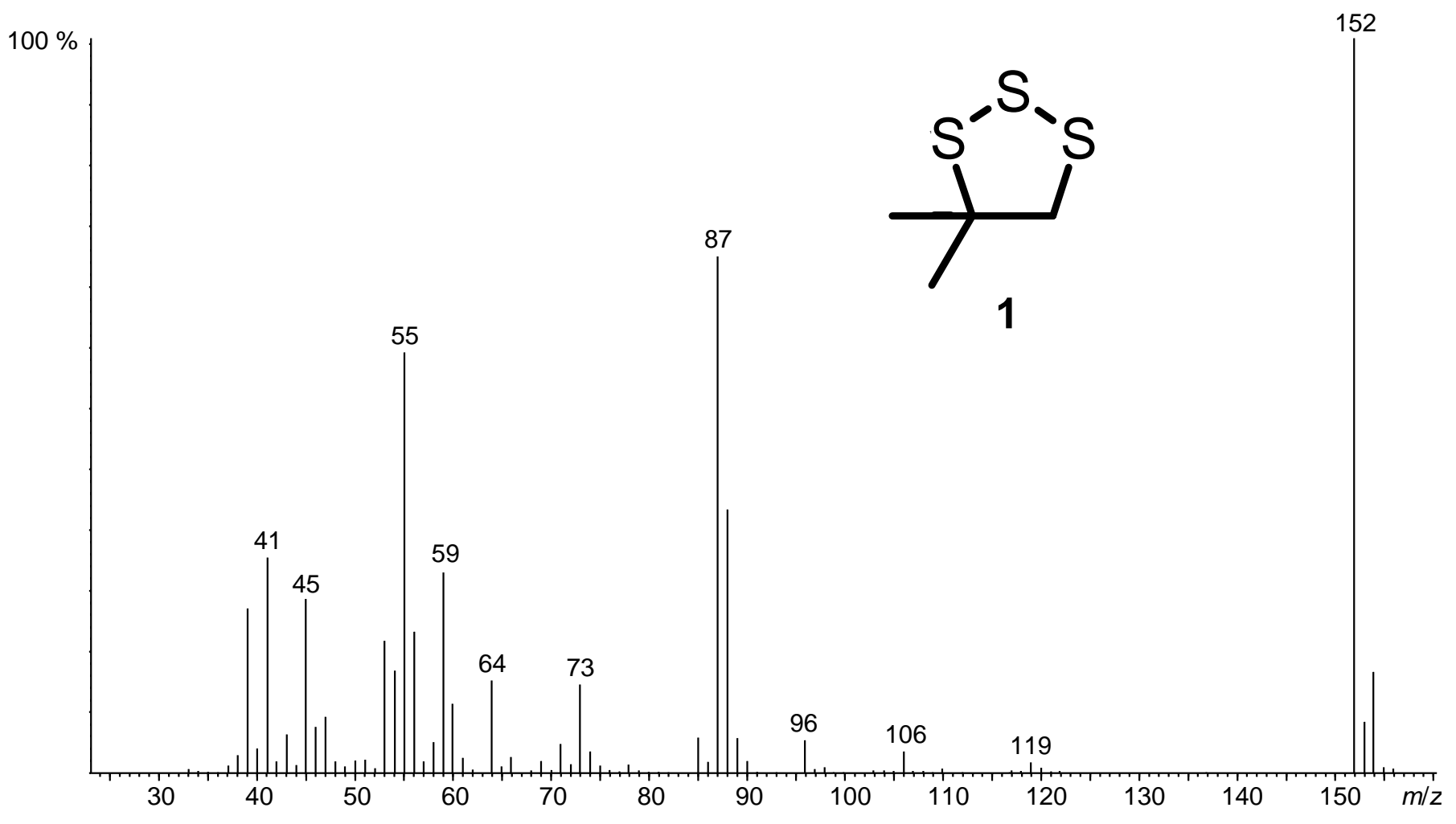

FIGURE S1. MS spectrum of 4,4-dimethyl-1,2,3-trithiolane (1). 


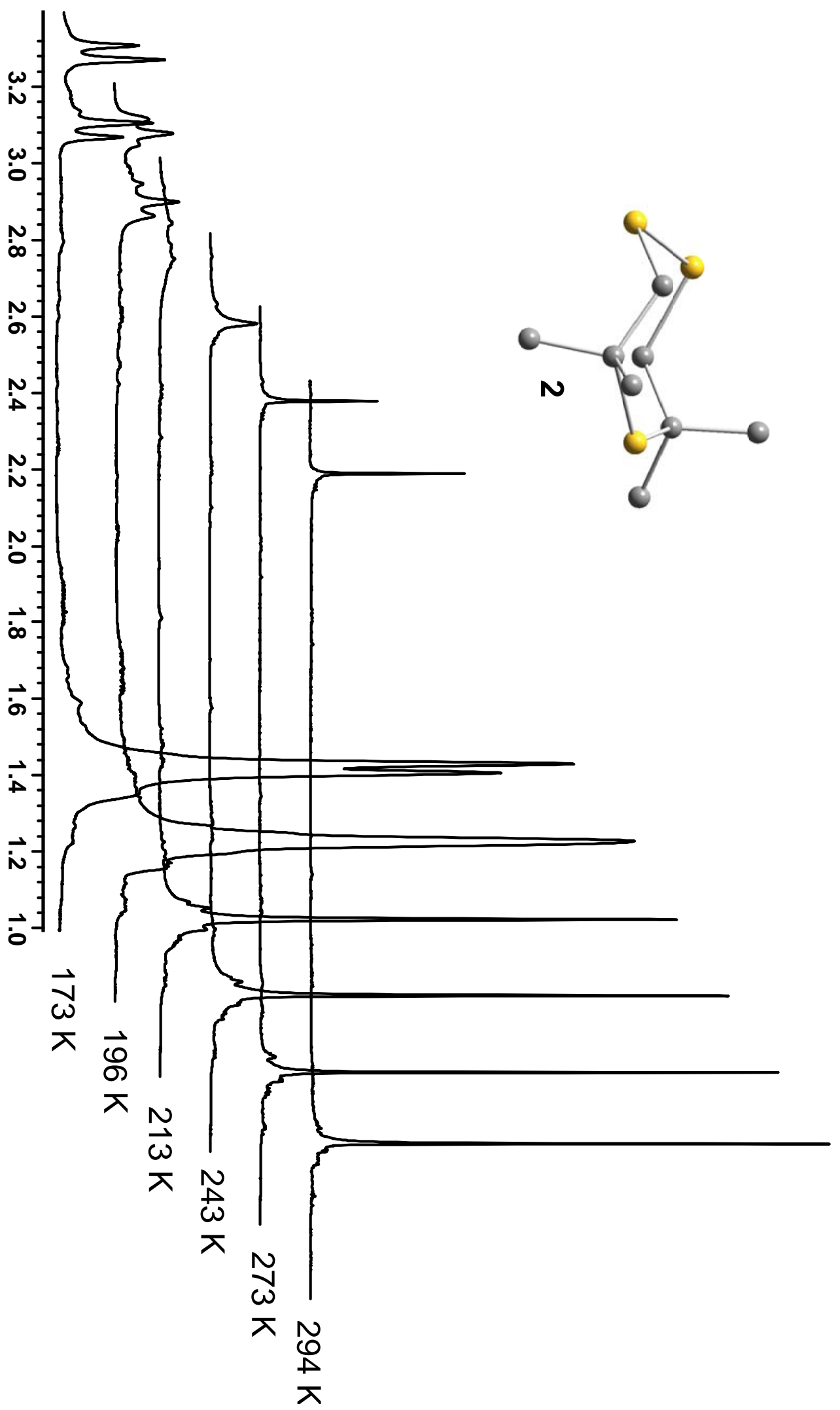

FIGURE S2. ${ }^{1} \mathrm{H}$-NMR spectra of 4,4,6,6-tetramethyl-1,2,5-trithiepane (2) at different temperatures. 


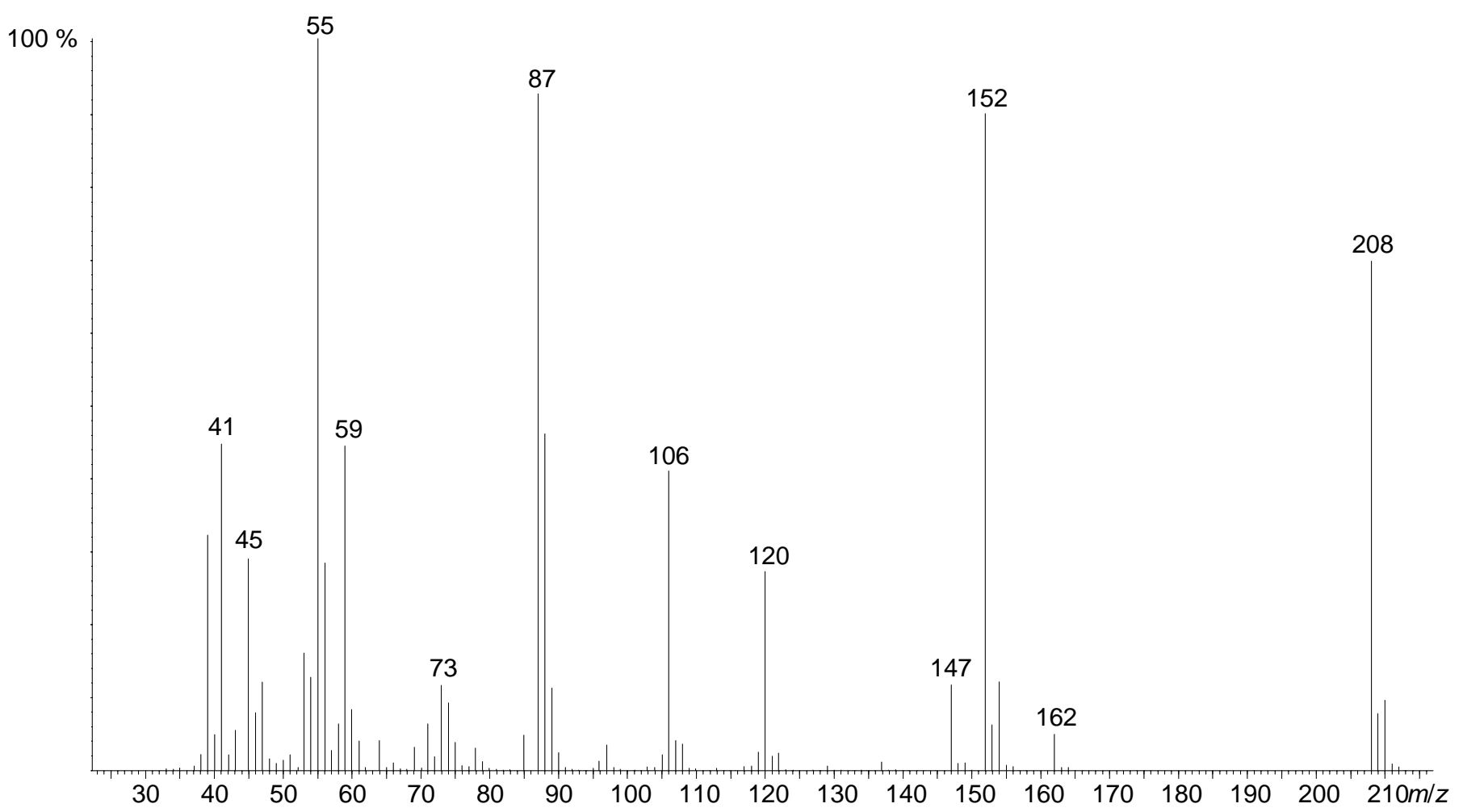

FIGURE S3. MS spectrum of 4,4,6,6-tetramethyl-1,2,5-trithiepane (2). 


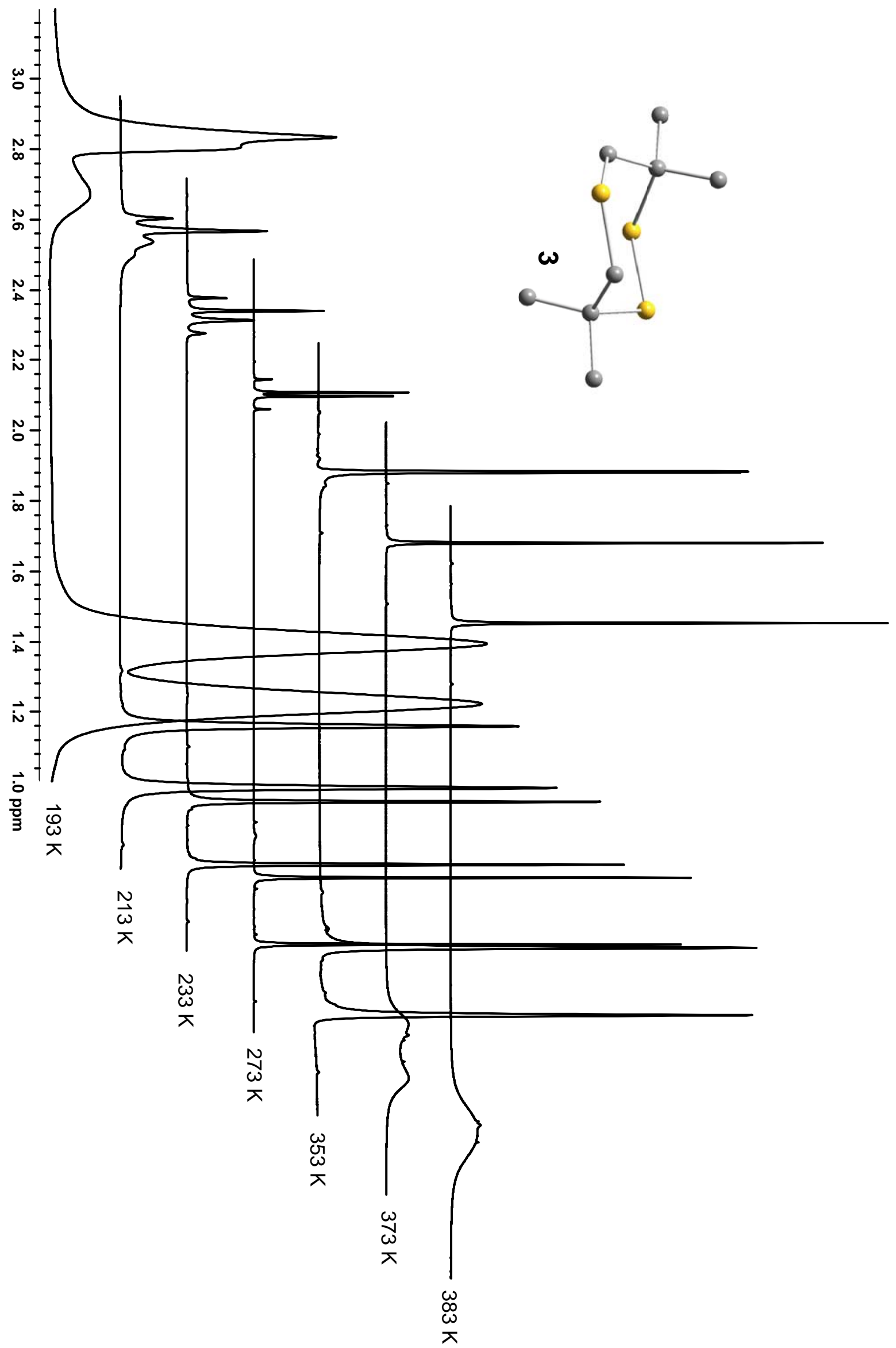

FIGURE S4. ${ }^{1}$ H-NMR spectra of 3,3,7,7-tetramethyl-1,2,5-trithiepane (3) at different temperatures. 


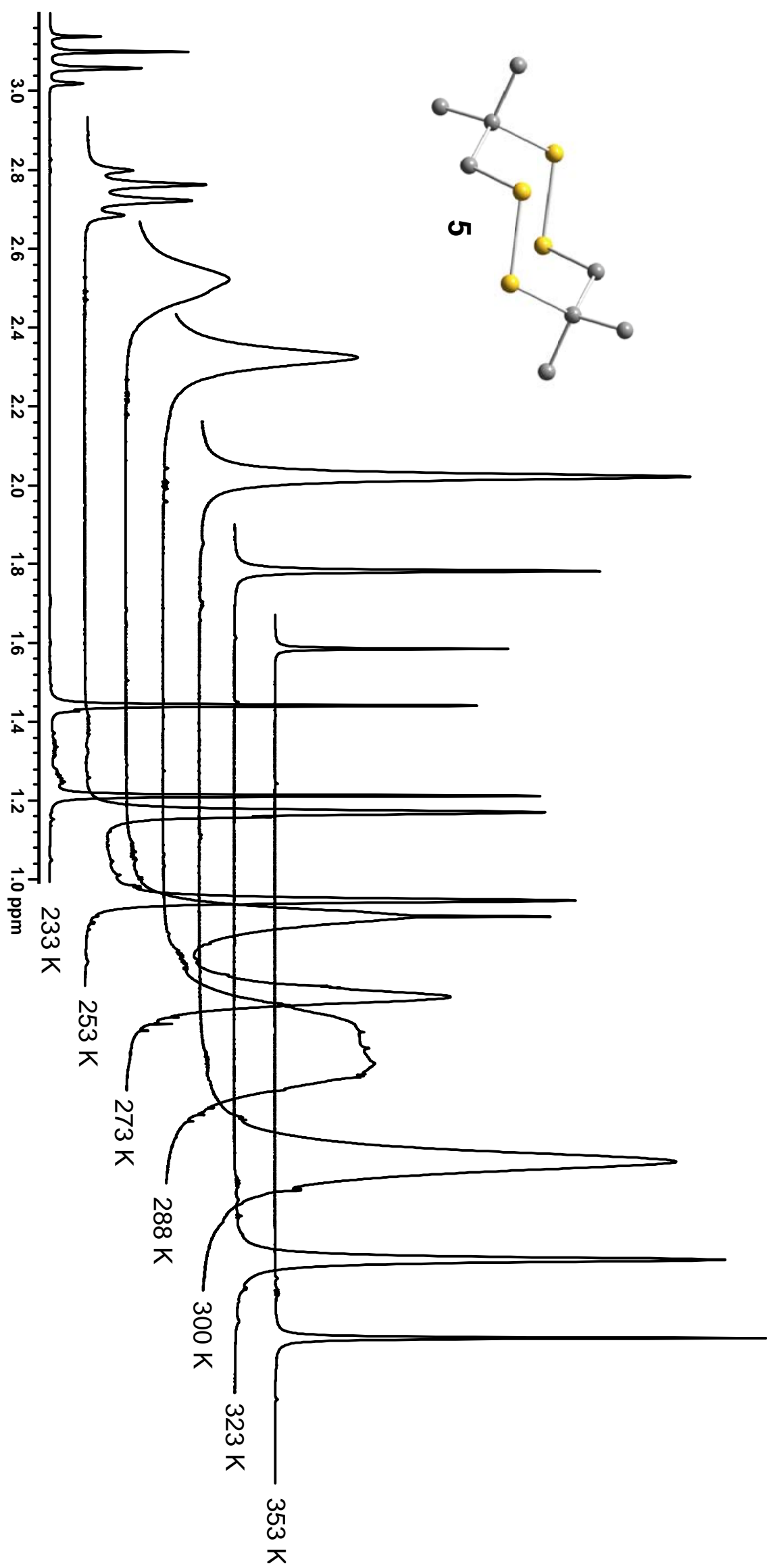

FIGURE S5. ${ }^{1} \mathrm{H}$-NMR spectra of 3,3,7,7-tetramethyl-1,2,5,6-tetrathiocane (5) at different temperatures. 


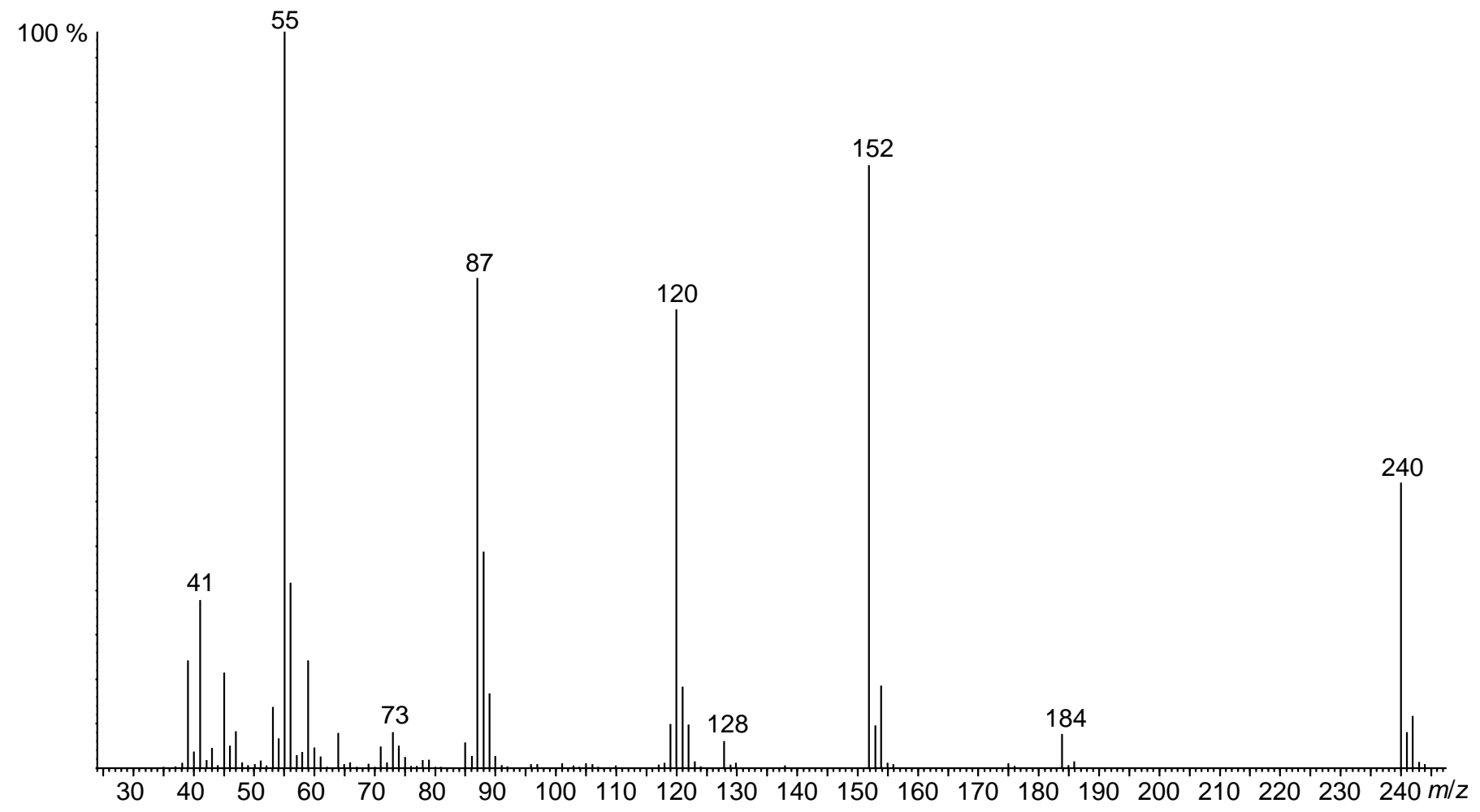

FIGURE S6. MS spectrum of 3,3,7,7-tetramethyl-1,2,5,6-tetrathiocane (5). 


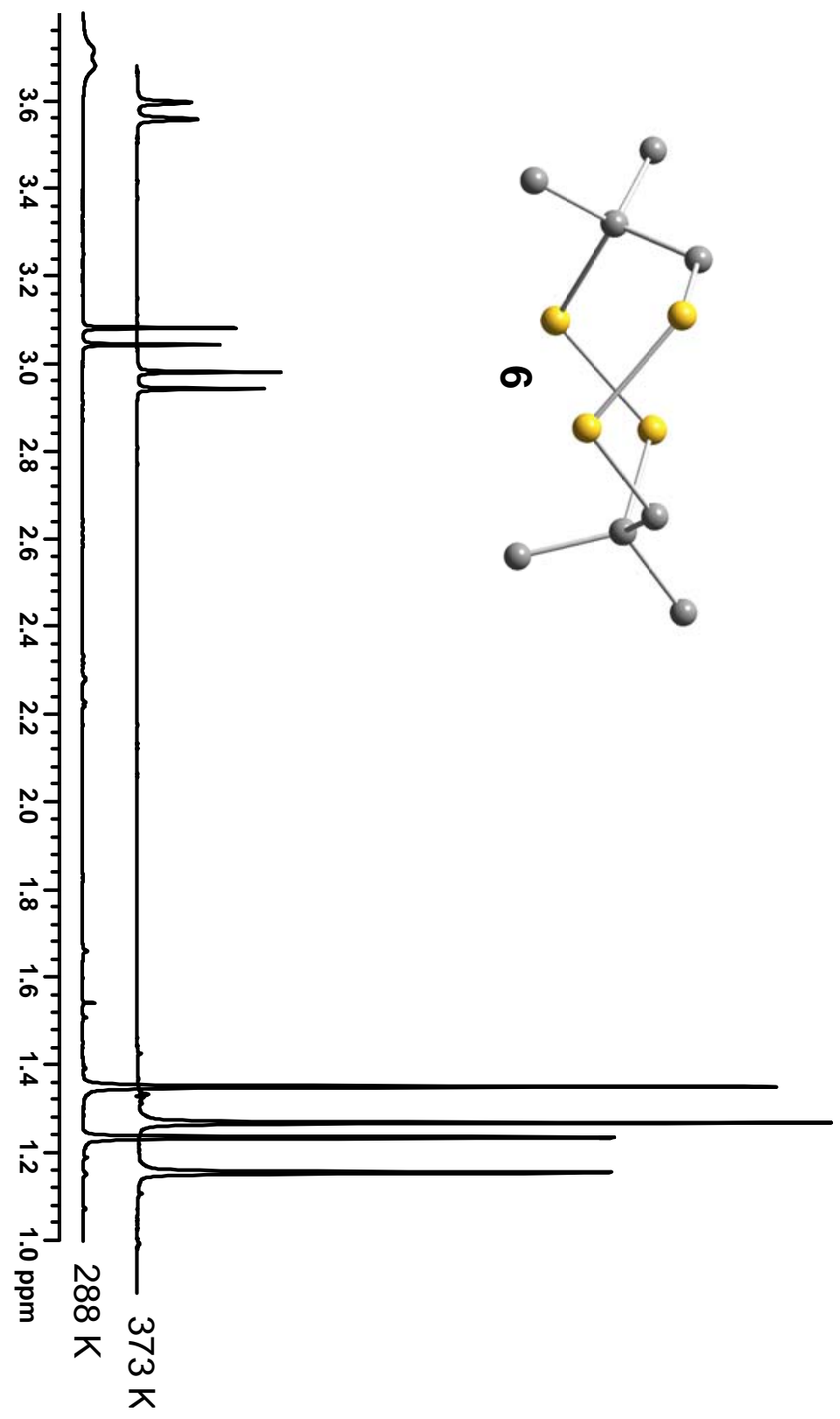

FIGURE S7. ${ }^{1} \mathrm{H}$-NMR spectra of 3,3,8,8-tetramethyl-1,2,5,6-tetrathiocane (6) at different temperatures. 


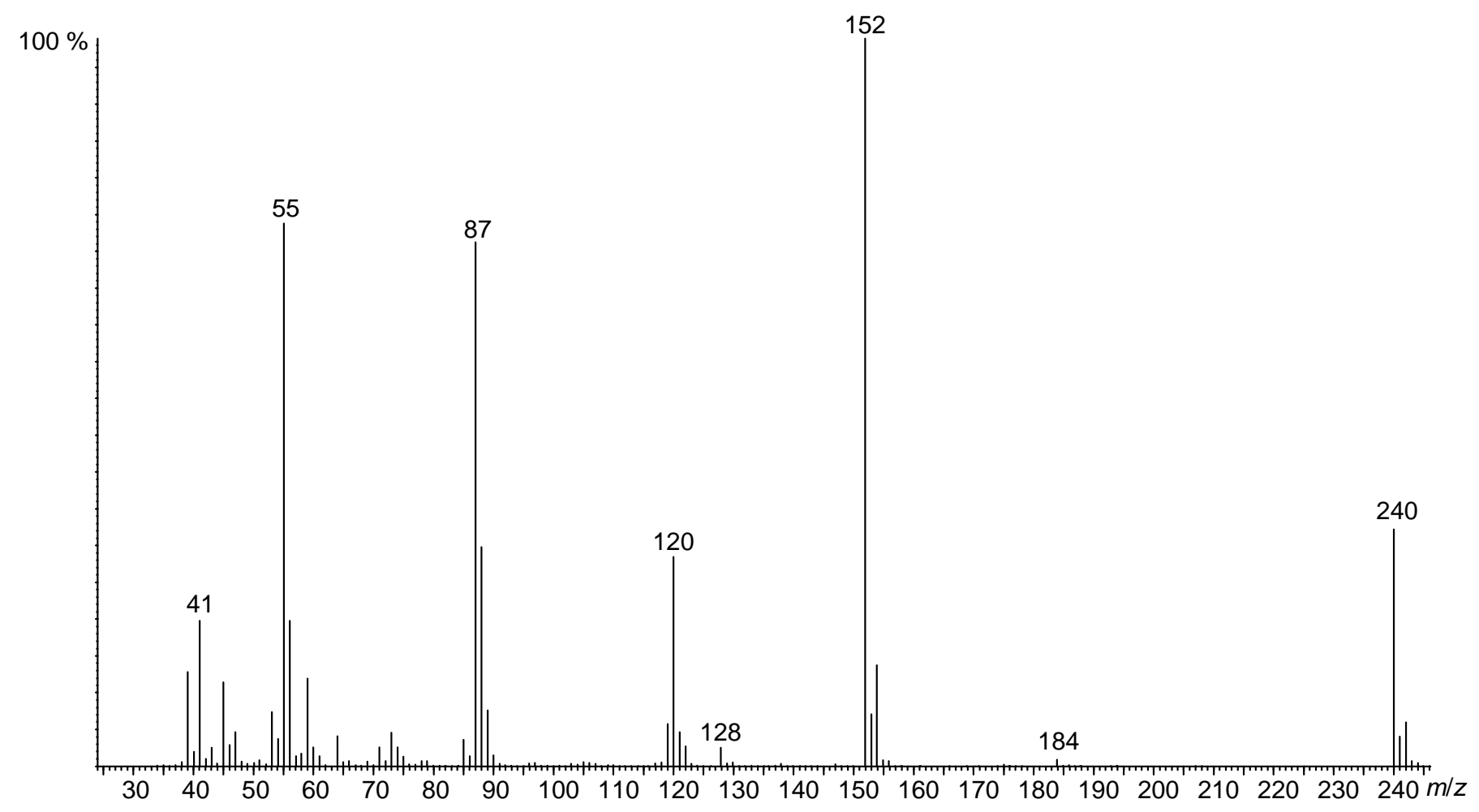

FIGURE S8. MS spectrum of 3,3,8,8-tetramethyl-1,2,5,6-tetrathiocane (6).

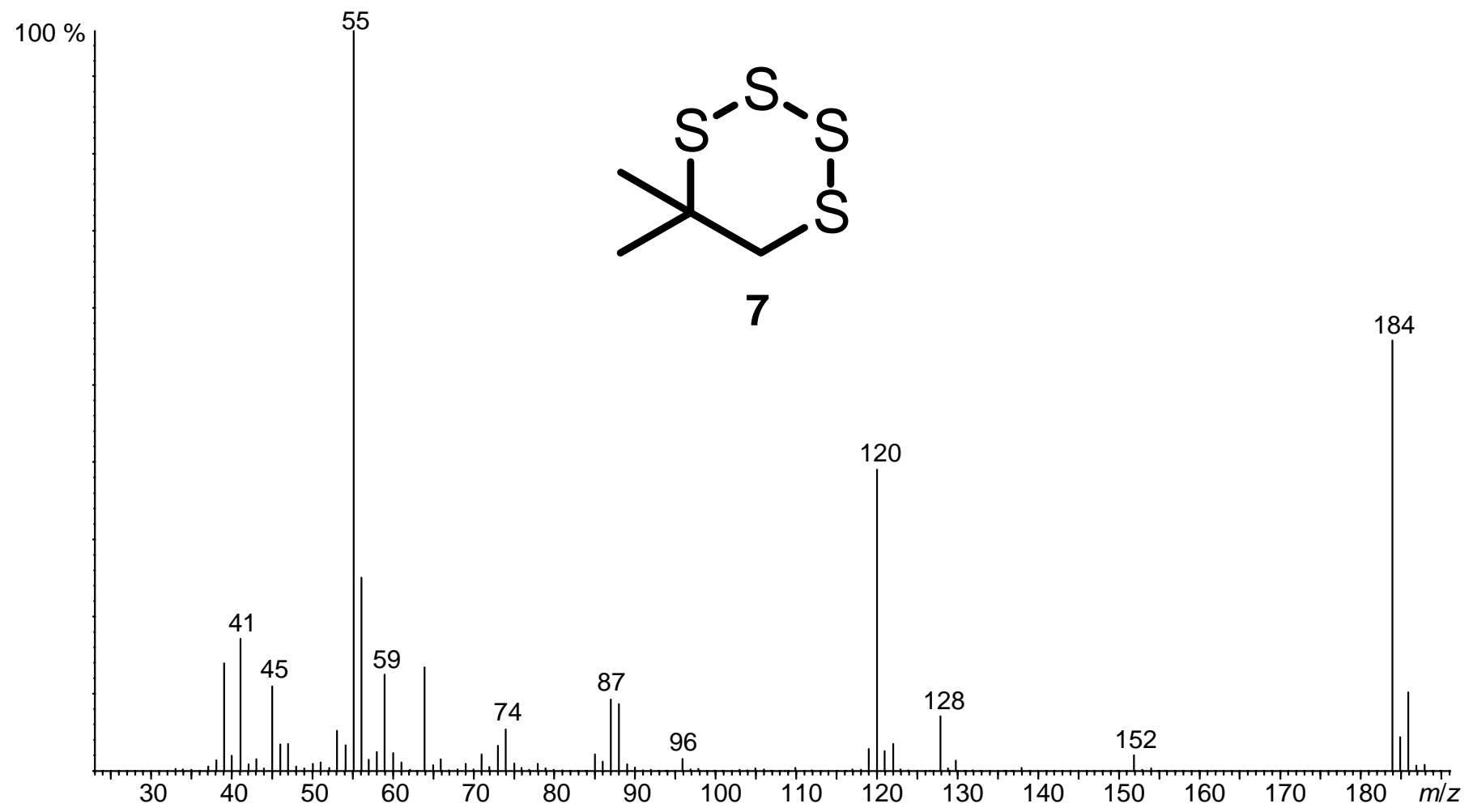

FIGURE S9. MS spectrum of 5,5-dimethyl-1,2,3,4-tetrathiane (7). 


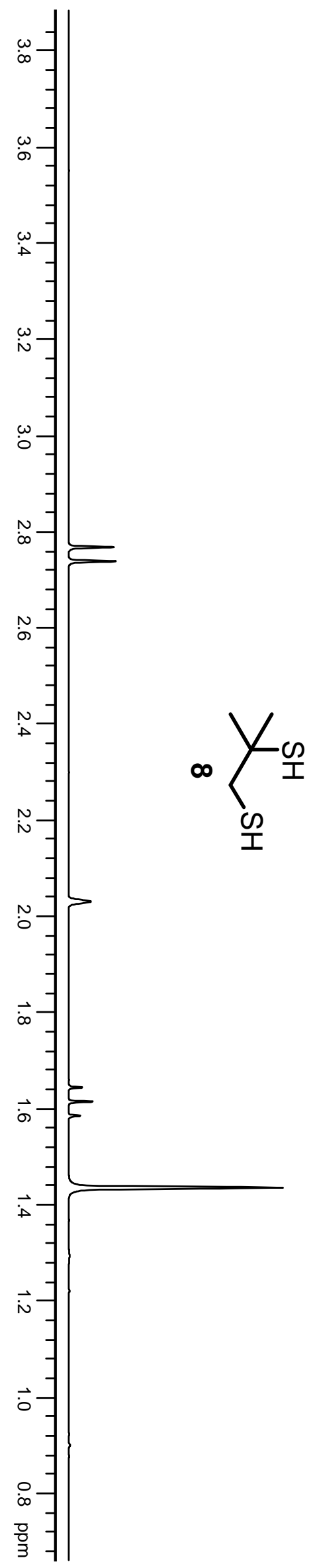

FIGURE S10. ${ }^{1}$ H-NMR spectrum of methyl-1,2-propanedithiol (8). 


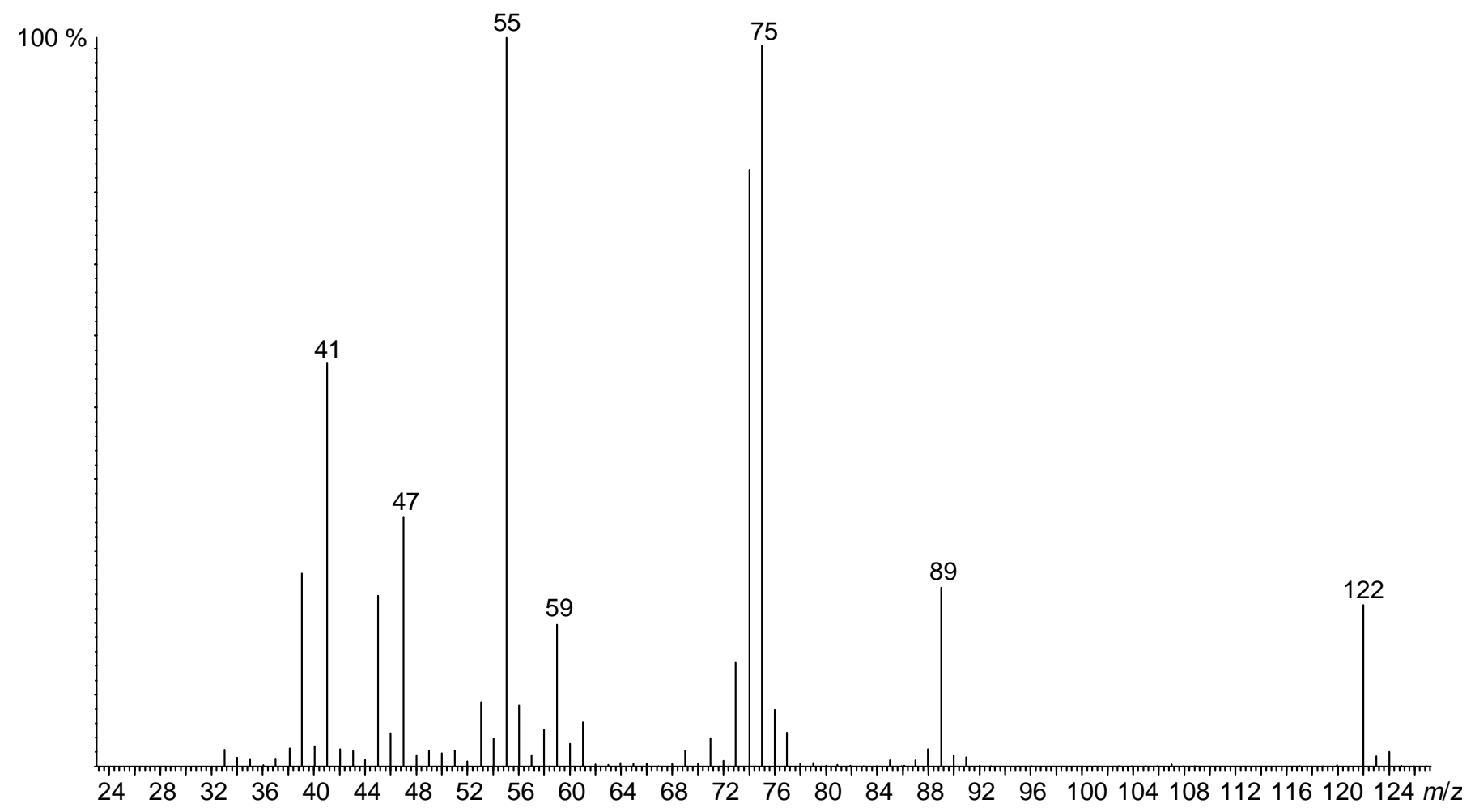

FIGURE S11. MS spectrum of methyl-1,2-propanedithiol (8). 


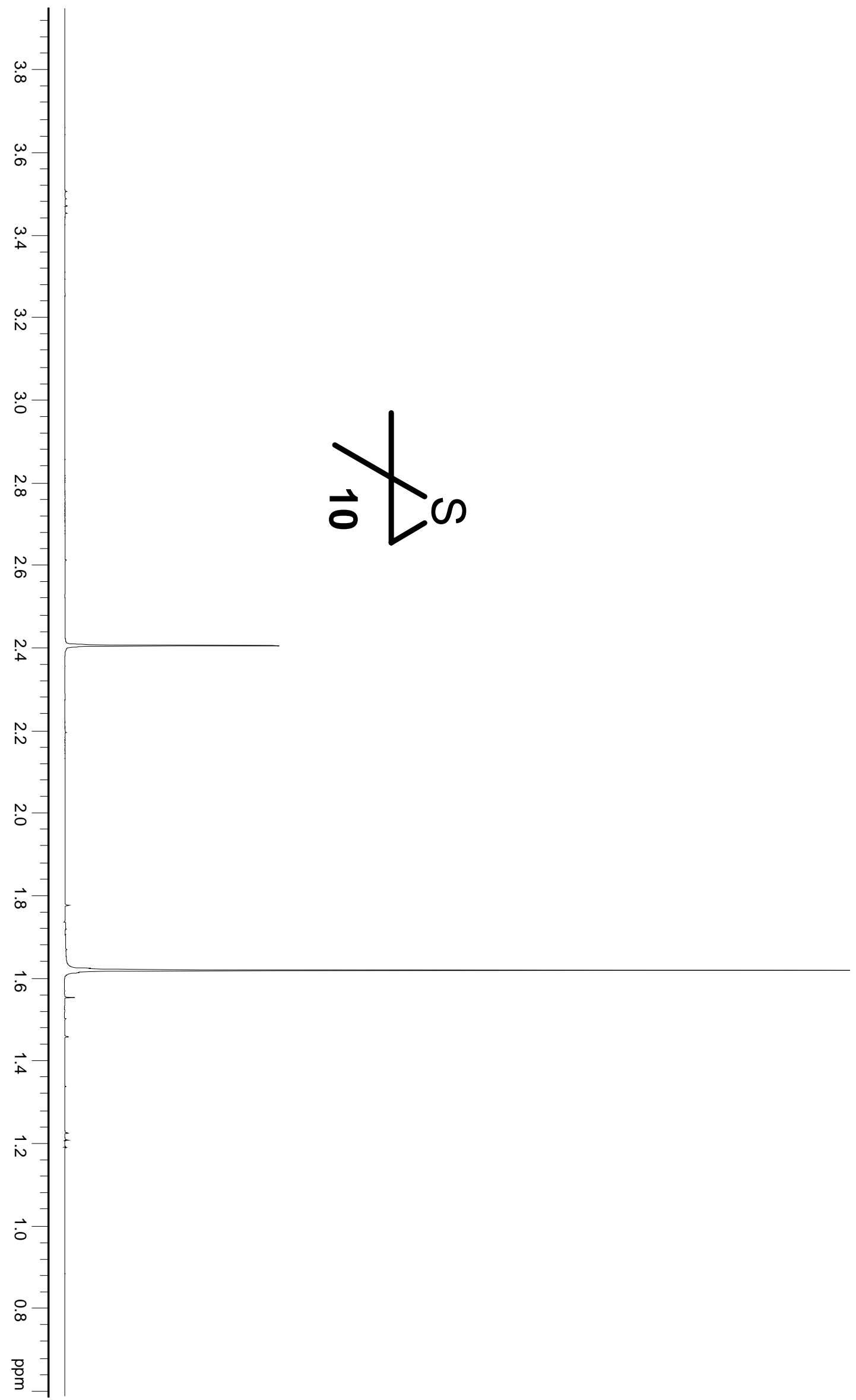

FIGURE S12. ${ }^{1} \mathrm{H}-\mathrm{NMR}$ spectrum of 2,2-dimethylthiirane (10). 


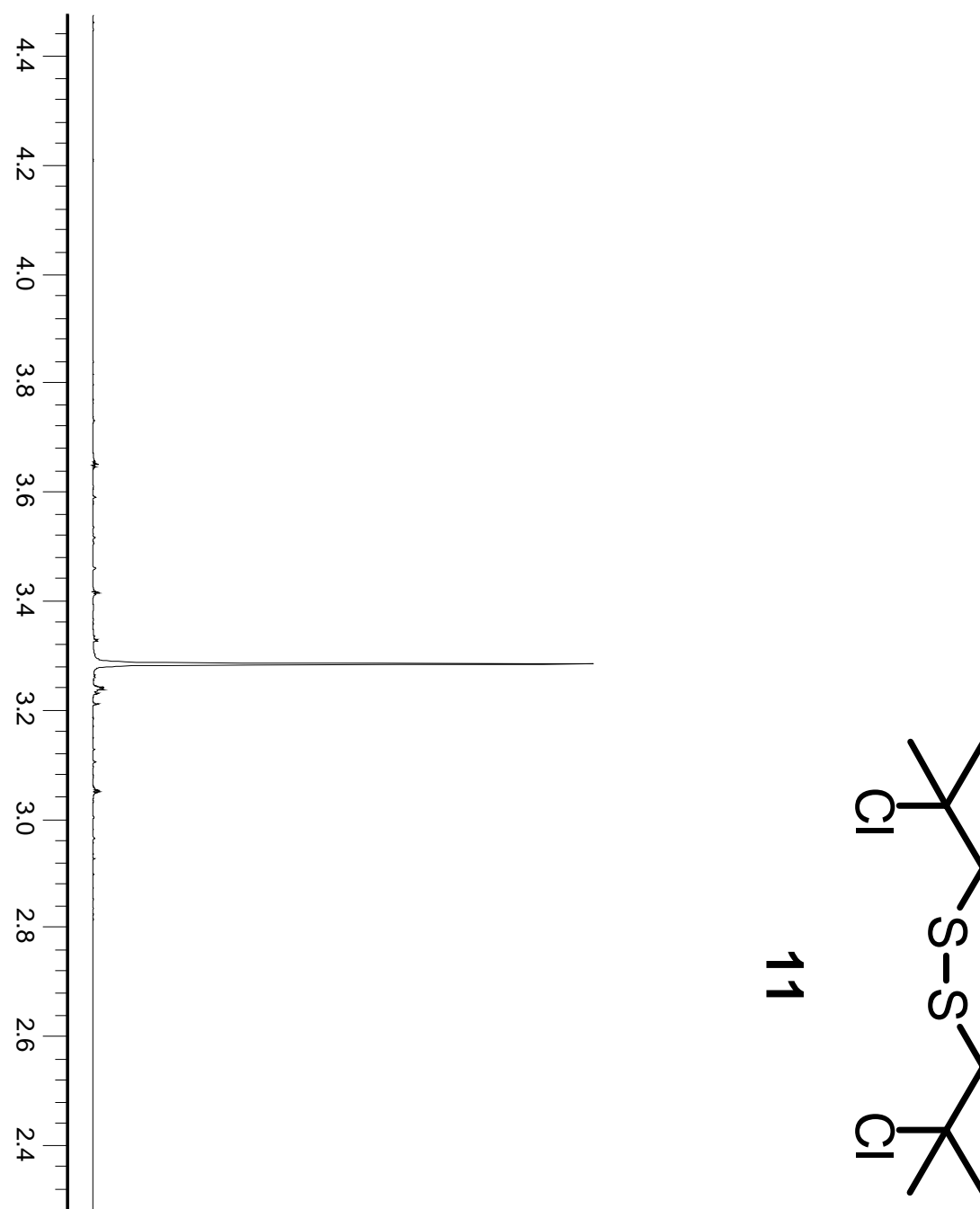

$\stackrel{N}{N}$

$\stackrel{0}{\circ}$

0

$\stackrel{+}{\infty}$

เ

ம்

$\stackrel{\bullet}{\oplus}$

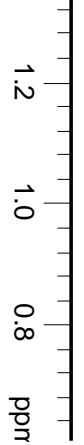

FIGURE S13. ${ }^{1}$ H-NMR spectrum of 2-chlor-1-(2-chlor-2-methylpropyldisulfanyl)-2-methylpropane (11). 


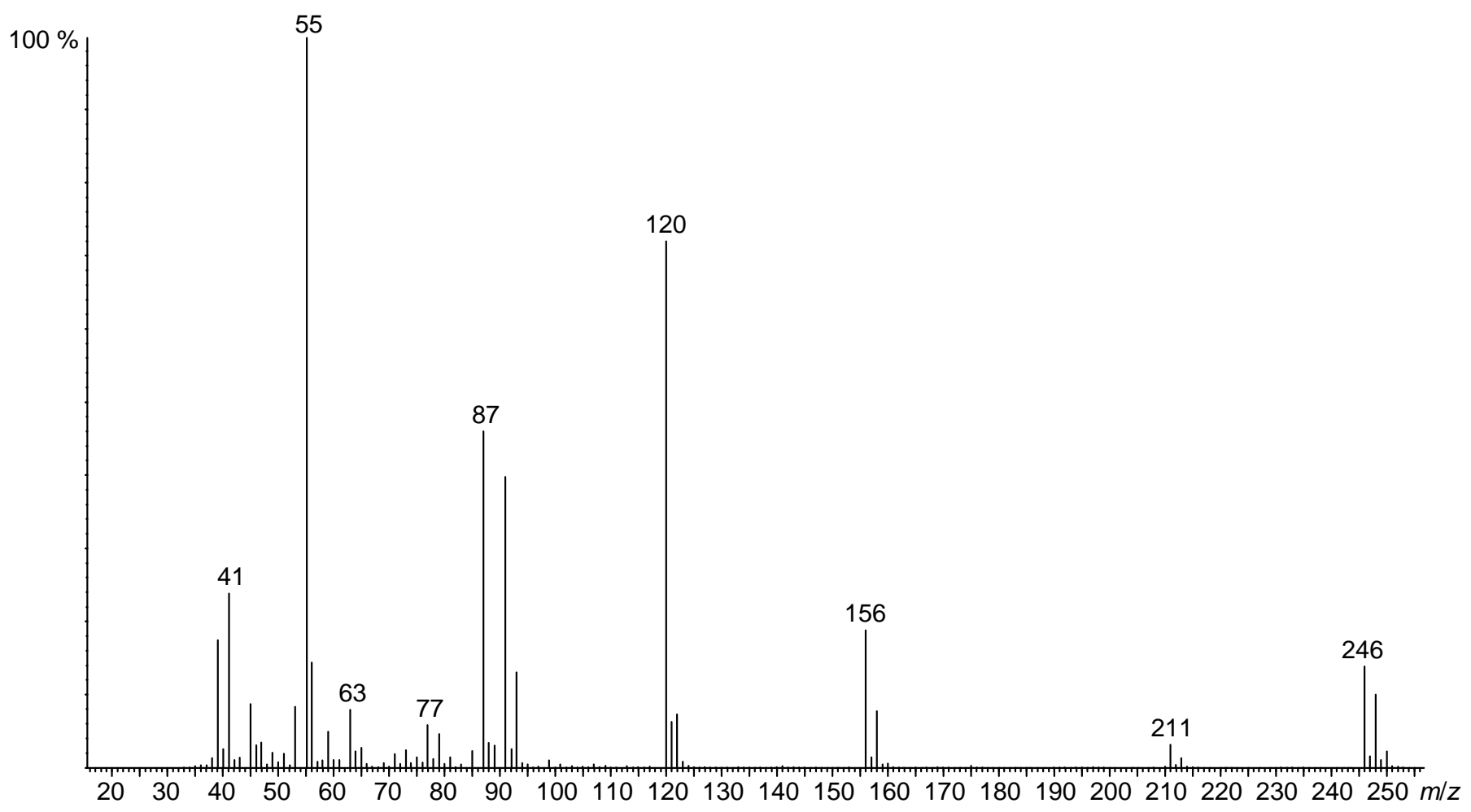

FIGURE S14. MS spectrum of 2-chlor-1-(2-chlor-2-methylpropyldisulfanyl)-2-methylpropane (11). 
TABLE S2: B3LYP 6-311G(d,p) optimized cartesian coordinates of all studied conformers for 3,3,6,6tetramethyl-1,2,5-trithiepane (4).

\section{twist-chair I}

C

$\mathrm{C}$

$\mathrm{C}$

$\mathrm{C}$

$\mathrm{H}$

$\mathrm{H}$

$\mathrm{H}$

$\mathrm{H}$

$\mathrm{S}$

$\mathrm{S}$

C

$\mathrm{H}$

$\mathrm{H}$

$\mathrm{H}$

C

$\mathrm{H}$

$\mathrm{H}$

$\mathrm{H}$

C

$\mathrm{H}$

$\mathrm{H}$

$\mathrm{H}$

C

$\mathrm{H}$

$\mathrm{H}$

$\mathrm{H}$

$\mathrm{S}$

\section{boat TS1}

C

C

C

C

$\mathrm{H}$

$\mathrm{H}$

$\mathrm{H}$

$\mathrm{H}$

$\mathrm{S}$

$\mathrm{S}$

C

$\mathrm{H}$

$\mathrm{H}$

$\mathrm{H}$

C

$\mathrm{H}$

C
$-1.90802400$
1. 57195300
$-0.67874400$
2. 01635100
$-0.32709100$
2. 37363100
1.39384000
$-1.00079300$
๑. 09035800
$-1.46703100$
$-2.91402000$
$-3.82014800$
$-3.19752300$
$-2.48880500$
$-2.54822800$
$-2.95168000$
$-3.37620500$
$-1.81840300$
2. 40353500
2. 73492400
3. 22510600
1.57888000
3. 22911700
4.04346800
3. 59285600
2.97765900
0.75346300

$-0.47271400$

1.17923700

$-1.12916400$

$-0.18852100$

$-0.51835700$

1. 90587900

1.12521200

$-2.08405000$

1.97663200

1.08529800

$-0.12177500$

$\odot .31572000$

$-1.02145400$

$\odot .59619000$

$-1.38418700$

$-2.28047300$

$-0.88111500$

$-1.68950000$

$-0.11463100$

$-1.08977900$

0.59878800

0.22605100

$-0.65099100$

0.07342400

$-1.61926400$

$-0.74109000$

$-1.53816300$
0.13997600

$-0.51114600$

0.79230000

0.03349000

1.62208200

$-0.33474300$

$-1.58477200$

1. 21803800

$\odot .24267900$

$-0.81693100$

1. 24546100

0.82192500

1.80352700

1.95066300

$-0.91541900$

$-0.43279900$

$-1.42261300$

$-1.66686800$

1. 51393400

1.87473200

1.64439000

2.14168000

$-0.79636100$

$-0.69897500$

$-0.44567600$

$-1.85590700$

$-0.27875300$
1.90287900

$-1.61251600$

0.74144400

$-2.01925200$

0.45312900

$-2.00718800$

$-2.10960400$

1.08634100

0.11250100

1. 29208800

3. 00263600

3.85673400

3. 35130800

2.63509600

2. 45773300

2. 91511700
$-0.46729500$

1. 32812300

$-1.01040600$

$-0.16420200$

$-0.28309900$

1.66671900

1. 91387700

$-1.90375200$

2. 00795700

0.91527300

0.05660100

0.42219400

$-0.74558400$

0.87344000

$-1.52223300$

$-2.33767900$
$-0.12477300$

$-0.20781100$

$-0.97750300$

0.00889100

$-1.73484200$

$-1.16926300$

0.56532400

$-1.50702300$

$-0.31914700$

0.99072800

$-1.05648300$

$-0.48303900$

$-1.71659700$

$-1.68178200$

0.83964300

$\odot .26999200$ 
$\mathrm{H}$
$\mathrm{H}$
$\mathrm{C}$
$\mathrm{H}$
$\mathrm{H}$
$\mathrm{H}$
$\mathrm{C}$
$\mathrm{H}$
$\mathrm{H}$
$\mathrm{H}$
$\mathrm{S}$

\section{twist boat}

C

C

C

C

$\mathrm{H}$

$\mathrm{H}$

$\mathrm{H}$

$\mathrm{H}$

$\mathrm{S}$

$\mathrm{S}$

C

$\mathrm{H}$

$\mathrm{H}$

$\mathrm{H}$

C

$\mathrm{H}$

$\mathrm{H}$

$\mathrm{H}$

C

$\mathrm{H}$

$\mathrm{H}$

$\mathrm{H}$

C

$\mathrm{H}$

$\mathrm{H}$

$\mathrm{H}$

$\mathrm{S}$

\section{boat TS2}

C

C

C

C

$\mathrm{H}$

$\mathrm{H}$

$\mathrm{H}$

$\mathrm{H}$
3.23056100

1. 66979800

- 3.09372900

- 3.48739600

$-3.92753900$

$-2.68111200$

$-2.60738800$

$-3.49136800$

- 2.91466400

$-1.87484300$

$-0.73804000$
$-1.09470000$

$-1.93740500$

$-0.50742100$

$-1.51248800$

๑ . 20186400

$-0.46744700$

$-0.31486100$

๑. 32247800

$-1.34634000$

$-0.03199800$

$-1.55635000$
1. 48428200

1.46977500

$-1.03961800$

$-0.87627400$

$-0.97913900$

$-2.05038200$

1. 42508800

1.53235200

1. 61541300

2.18256300

$-0.05445400$
1.87944400

$-1.49252700$

0.75856400

$-1.93929400$

๑. 48163500

$-1.35224300$

$-2.30312500$

1. 14922700

0.00161500

1. 17647200

2.95735900

3. 79403800

3. 34063000

2.55721600

2.47788500

2. 98334800

3. 21901100

1. 70404900

- 3.26006300

$-3.58154200$

$-4.05052900$

- 3.14970000

$-2.17549500$

$-2.90402600$

$-2.58216300$

$-1.26050800$

$-0.75812100$
$-0.44305500$

1. 02936200

$-1.08793100$

$-0.17570400$

$-0.42843100$

0.72564500

1.76372500

- 2 . 00214000

2. 05107800

$\odot .91937900$

๑. 10482200

$\odot .51605600$

$-0.69498400$

$\odot .89383500$

$-1.43493100$

$-2.24157100$

$-0.94887800$

$-1.87336600$

$-0.68433600$

$-1.60369800$

0.06227800

- $\odot .89892700$

$\odot .17482500$

$\odot .98991800$

$-0.68941000$

0.48007100

$-1.65473500$
$-0.10366700$

$-0.79544200$

$-0.94155100$

0.04143300

$-1.76379200$

$-1.83384200$

$-0.77431800$

$-1.39968200$

$-0.33817600$

0.96176100

$-1.04605300$

$-0.47796600$

$-1.68966500$

$-1.68668000$

0.90244400

๑. 36175300

1.54233400

1.53440800

$-0.57269700$

$-0.07789200$

$-0.44204000$

$-1.63727800$

1.51636100

1.58974700

2. 04676500

2.01760900

$-0.08073700$
$-1.90384800$

1.37214300

$-0.65741800$

1. 99263000

$-0.24916200$

$\odot .82177400$

2.18827000

$-0.99750000$
$-0.50462200$

0.85133300

$-1.37731000$

$-0.30530800$

$-1.19124500$

$\odot .48923800$

1.46709700

$-2.41652400$
0.14056400

$\odot .91521900$

๑. 38668300

0.10759700

1. 37918700

1.78360500

1.31146400

$\odot .39035800$ 


\begin{tabular}{|c|c|c|}
\hline ๑.3692790९ & 2.08737300 & $\odot .02503900$ \\
\hline-1.61277700 & 1. 29519100 & -0.36500300 \\
\hline-2.75176200 & $-\odot .5 \odot 21970 \odot$ & $1.4229070 \odot$ \\
\hline-3.68212800 & ๑. 04891500 & 1.27549800 \\
\hline-3.00666800 & -1.52995400 & 1.707572 \\
\hline-2.21464100 & -0.03651400 & 2.25157100 \\
\hline-2.71338800 & -1.06190700 & -1.0411340 \\
\hline-3.09406700 & -2.05754000 & -0.78619400 \\
\hline-3.57190100 & -0.42609500 & -1.27114000 \\
\hline-2.09524700 & -1.13509600 & -1.93697800 \\
\hline 2.73441300 & -1.22981800 & 1.0869180 \\
\hline 3.22473300 & -2.04234500 & $\odot .5483790 \odot$ \\
\hline 3.49990300 & $-\odot .6706990 \odot$ & 1.63730200 \\
\hline 2.05418500 & -1.67407300 & 1.81820900 \\
\hline $2.9537950 \odot$ & 0.21763800 & $-\odot .9664300 \odot$ \\
\hline 3.80570700 & ๑. 71585300 & $-\odot .4916540$ \\
\hline 3.34303100 & $-0.6041940 \odot$ & -1.57259400 \\
\hline 2.45557100 & $\odot .93015200$ & -1.62454200 \\
\hline$\odot .7034200$ & -1.28217900 & $-\odot .830910 €$ \\
\hline
\end{tabular}

\section{chair}

C

C

C

C

$\mathrm{H}$

$\mathrm{H}$

$\mathrm{H}$

$\mathrm{H}$

$\mathrm{S}$

$\mathrm{S}$

C

$\mathrm{H}$

$\mathrm{H}$

$\mathrm{H}$

C

$\mathrm{H}$

$\mathrm{H}$

$\mathrm{H}$

C

$\mathrm{H}$

$\mathrm{H}$

$\mathrm{H}$

C

$\mathrm{H}$

$\mathrm{H}$

$\mathrm{H}$

$\mathrm{S}$

\begin{abstract}
1.87842100
$-1.37725300$

0.69334200

$-2.02504700$

$\odot .43935900$

- 0.90197000

$-2.17032600$

1. 02588300

$-0.11743000$

1.64953800

3. 12221800

3. 97685900

3. 39387100

2. 94130700

2. 14742700

2. 27997200

3. 05481500

1.31580900

$-2.77844900$

$-3.32528400$

$-3.49720700$

$-2.09267300$

$-2.99212700$

$-3.82081000$

$-3.41468300$

$-2.48588300$

$-0.82774700$
\end{abstract}

$-0.52214000$

1.12708900

$-1.40096900$

$-0.18365700$

$-1.18148000$

0.99362400

1.87137800

$-2.44343400$

1. 90263900

1. 27246700

$-0.93227300$

$-0.29491900$

$-1.96308600$

$-0.87547900$

$-0.64735900$

$-1.70070700$

$-0.10353000$

$-0.25445300$

$-0.81439900$

$-1.70267000$

$-0.10136300$

$-1.10915500$

0.10022100

0.72226900

$-0.82598500$

0.62153300

$-1.42583200$ $\odot .00345600$

$-0.65141100$

$-0.46672400$

$-0.16124500$

$-1.50505500$

$-1.62336600$

$-0.77787400$

$-0.43916100$

0.44037100

$-0.48129600$

$-0.80777600$

$-0.56850800$

$-0.56083200$

$-1.88416700$

1.50259900

1.77496900

1.77243300

2. 08721700

$-1.34359000$

$-1.02277500$

$-1.76401000$

$-2.14176900$

0.99880900

0.64552700

1. 39634100

1.81366000

$\odot .56296800$ 
$\mathrm{S}$
$\mathrm{C}$
$\mathrm{S}$
$\mathrm{S}$
$\mathrm{C}$
$\mathrm{H}$
$\mathrm{H}$
$\mathrm{C}$
$\mathrm{H}$
$\mathrm{H}$
$\mathrm{H}$
$\mathrm{C}$
$\mathrm{H}$
$\mathrm{H}$
$\mathrm{H}$
$\mathrm{C}$
$\mathrm{H}$
$\mathrm{H}$
$\mathrm{H}$
$\mathrm{C}$
$\mathrm{H}$
$\mathrm{H}$
$\mathrm{H}$
$\mathrm{C}$
$\mathrm{C}$
$\mathrm{H}$
$\mathrm{H}$

C

$\mathrm{H}$

$\mathrm{H}$

$\mathrm{H}$

C

$\mathrm{H}$

H

$\mathrm{H}$

C

$\mathrm{H}$

$\mathrm{H}$

$\mathrm{H}$

C

$\mathrm{H}$

$\mathrm{H}$

$\mathrm{H}$

C

C

$\mathrm{H}$

$\mathrm{H}$

\section{twist-chair II}

C

C

$\mathrm{S}$

C

$\mathrm{H}$

$\mathrm{H}$

$\mathrm{S}$

$S$

C

$\mathrm{H}$

$\mathrm{H}$

C

$\mathrm{H}$

$\mathrm{H}$

$\mathrm{H}$

C

$\mathrm{H}$

$\mathrm{H}$

$\mathrm{H}$

C

$\mathrm{H}$

$\mathrm{H}$
$-0.95647700$

$-1.94942100$

1.56752700

$-0.04059300$

$-1.50422000$

$-2.30539800$

$-1.34614700$

1. 76211800

2. 49653300

0.78243300

1.98128500

3. 27524000

3. 99039400

3. 51748000

3. 40013100

- 2.09077300

$-2.79119200$

$-2.47939100$

$-1.14739300$

$-3.32792200$

$-4.06214100$

- 3.29621200

$-3.67095200$

1. 83374800

0.91435100

1. 20187300

1. 12266400
$-1.63996100$

$-0.13415000$

1.08369700

2. 03690000

1.18531500

1.92175200

1.05350700

$-0.29428800$

๑ . 44806000

0.04488600

$-1.23849300$

$-0.90185700$

$-0.17398600$

$-1.88101100$

$-0.96535300$

$-0.03496200$

0.76883600

$-0.97079000$

$\odot .18709500$

$-0.48962900$

๑. 25826400

$-0.51030500$

$-1.46525100$

$-0.50524100$

$-1.62631500$

$-1.84552400$

$-2.51510000$
$-0.46074500$

$\odot .08198700$

$-0.77872800$

$\odot .13138500$

$-0.56973100$

$-0.43290100$

$-1.64007200$

1. 69243700

2. 01134100

2. 01841600

2. 20138600

$-0.20779500$

$\odot .17964600$

$\odot .21589800$

$-1.29197500$

1. 60388400

1.85971100

2 . 00813400

2. 09690300

$-0.52079800$

- $\odot .20631200$

$-1.61169200$

$-0.16894200$

$\odot .18143700$

$-0.33154200$

$-1.36024400$

๑. 27081800
1.90791200

0.67860300

$-0.75338000$

$-2.01609100$

1. 00074300

๑. 32654400

1. 46619900

$-0.09032300$

$-1.57277100$

$-2.37431400$

$-1.39628700$

2. 91143100

3. 81738100

2.48362100

3. 19580700

2. 55131600

3. 37964200

2. 95496500

1. 82341900

- 2.40097700

$-3.22181600$

$-2.73250900$
$-0.47263100$

$-1.13091800$

$-1.53781100$

$-0.18867900$

$-2.08667800$

$-\odot .52208600$

1. 08457100

1.97688600

1.17968900

1. 90600400

1.12684300

$-0.12012700$

$\odot .31934900$

0.59660600

$-1.01950400$

$-1.38310700$

$-0.87883400$

$-2.27898100$

$-1.68922000$

$-0.11649100$

0.59732400

$-1.09192300$
0.14030100

$\odot .79068300$

$-0.28141100$

๑ . 03386800

1. 21439900

1.62172200

$-0.81771600$

$\odot .24226500$

$-0.51007300$

$-0.33171400$

$-1.58401500$

1. 24749400

$\odot .82566700$

1. 95237300

1.80562500

$-0.91395500$

$-1.41938500$

$-0.43075700$

$-1.66694800$

1.51498700

1.64776300

1.87489200 
$-1.57512700 \quad 0.22265600$

2.14198100

$\mathrm{C}$

$-3.23010400 \quad-0.65034500$

$-0.79458800$

$-4.04502300$

0.07291200

$-\odot .69341600$

$\mathrm{H}$

$-2.98088700$

$-0.73719600$

$-1.85492200$

$\mathrm{H}$

$-3.59201600$

$-1.61996100$

$-0.44577300$ 